\title{
Noninvasive evaluation of intracranial hypertension? Is there a gold standard?
}

\author{
Leonardo Christian Welling ${ }^{1}$, Eberval Gadelha Figueiredo², Fábio Santana Machado², \\ Almir Ferreira Andrade ${ }^{4}$, Vinicius Monteiro Guirado ${ }^{5}$, Manoel Jacobsen Teixeira ${ }^{6}$ \\ Division of Neurological Surgery, Hospital Bom Jesus, Ponta Grossa, PR, Brazil. \\ Division of Neurological Surgery, Hospital das Clinicas, University of Sao Paulo, SP, Brazil. \\ Neurointensive Care Post Graduation Program, Hospital Sírio-Libanês, SP, Brazil.
}

\section{ABSTRACT}

Computed tomography is essential in head injuried patients for the detection of structural damage to the brain. However, the ability of CT scanning to predict the presence or absence of intracranial hypertension has been debated in the literature. Since the optic nerve is part of the central nervous system and in case of raised pressure in the cerebrospinal fluid its sheath inflates. Based in this hypothesis the authors reviewed the role of the optic nerve sheat diameter in diagnosis intracranial hypertension after traumatic brain injury. This non-invasive method is useful to predict the risk of intracranial hypertension and select patients to ICP monitoring, especially in those with normal CT scans.

\section{KEYWORDS}

Intracranial hypertension, optic nerve injuries, tomography.

\section{RESUMO}

Avaliação não invasiva da hipertensão intracraniana? Existe um "padrão-ouro"?

A tomografia de crânio é um exame fundamental para avaliação estrutural dos pacientes com traumatismo craniano. Entretanto, a capacidade da tomografia em predizer a ausência ou presença de hipertensão intracraniana é controversa. Como o nervo óptico é parte do sistema nervoso central, em situações de hipertensão intracraniana há aumento da bainha neural. Com base nessas hipóteses, os autores revisaram o papel da análise do diâmetro da bainha do nervo óptico no diagnóstico de hipertensão intracraniana pós-traumatismo. Esse método não invasivo é útil para predizer os riscos de hipertensão intracraniana e selecionar os pacientes para monitorização da pressão intracraniana, principalmente naqueles com tomografia de crânio normal.

\section{PALAVRAS-CHAVE}

Hipertensão intracraniana, traumatismos do nervo óptico, tomografia.

1 Neurosurgeon, Division of Neurological Surgery, Hospital Bom Jesus, Ponta Grossa, PR, Neurointensive Care Post Graduation Program, Hospital Sírio-Libanês, Sao Paulo, SP, Brazil.

2 Head of Neurovascular Group and Supervisor of Division of Neurosurgery of Hospital das Clinicas, University of Sao Paulo, Sao Paulo, SP, Brazil.

3 Intensivist, coordinator of Neurointensive Post Graduation Program, Hospital Sírio-Libanês, Sao Paulo, SP, Brazil.

4 Head of Neurotraumatology Group of Division of Neurosurgery of Hospital das Clinicas, University of São Paulo, Sao Paulo, SP, Brazil.

5 Neurosurgeon, Division of Neurosurgery of Hospital das Clinicas, University of São Paulo, São Paulo, SP, Brazil.

6 Chairman, Division of Neurosurgery Hospital das Clinicas, University of Sao Paulo, São Paulo, SP, Brazil. 


\section{Introduction}

Traumatic brain injury (TBI) is responsible for up to $45 \%$ of in-hospital trauma mortality. ${ }^{1}$ Computed tomography (CT) is central to acute TBI diagnostics, and millions of brain CT scans are conducted yearly worldwide. ${ }^{2}$ This exam is valuable for the detection of structural damage to the brain. In traumatic brain injury and elevated intracranial pressure (ICP), invasive monitoring is used to guide treatment targeted at maintaining an adequate cerebral perfusion pressure (CPP). ${ }^{3}$

There is insufficient evidence to support standard of care level indications for invasive ICP monitor insertion..$^{4-8}$ The Brain Trauma Foundation guidelines recommend insertion for a Glasgow Coma Scale of 8 or less with computed tomography (CT) abnormality (hematoma, contusion, edema, or compressed basal cisterns) or a normal CT with two or more risk factors (older than 40 years old, motor posturing or systolic blood pressure less than $90 \mathrm{mmHg})^{3}$

According to Kishore et al., 17\% of the patients with "normal" CT scan at admission will develop intracranial hypertension. On the other hand many patients with abnormal CT scan do not develop intracranial hypertension. ${ }^{9}$ Raised ICP requires a rapid recognition to allow for the adequate treatment. The ability to predict whether or not ICP is elevated on the basis of CT scan findings would aid in defining which patients would benefit from more invasive monitoring, allow for more rapid treatment, and conserve precious neurosurgical resources. ${ }^{5,6}$ Based in this hypothesis the authors reviewed the role of the optic nerve sheat diameter in diagnosis intracranial hypertension in patients with severe traumatic brain injury.

\section{Anatomical considerations}

The optic nerves are phylogenetically an evagination of the brain. The course of the optic nerve can be subdivided into an intraocular, an intraorbital, a canalicular, and an intracranial segment. ${ }^{10}$ About $4 \mathrm{~mm}$ nasal to the central posterior pole of the globe, the nerve fibers of the inner ocular superficial layer converge and pierce the outer retina, the choroid, and the lamina crivosa, where they form the optic nerve head and the intraocular segment of the optic nerve. The mildly tortuous intraorbital optic nerve is about $4-4.5 \mathrm{~cm}$ long. It runs posteromedially in the center of the muscle cone to the orbital apex, where it enters the optic canal. The optic canal is about $5 \mathrm{~mm}$ long and $3-4 \mathrm{~mm}$ wide. ${ }^{10,11}$ The entire intraorbital and canalicular optic nerve is surrounded by arachnoid and dural sheaths, which enclose a tube-shaped subarachnoid space (optic nerve sheat - ONS). These sheaths and this space are extensions of the corresponding intracranial structures. The pia mater that envelops the optic nerve covers the retinal vessels as well. A slight ampullary bulging of the subarachnoid space of the anterior intraocular segment is normal. ${ }^{10,11}$ In patients with intracranial hypertension, the greatest degree of widening is usually seen here. A gradient of CSF pressure has been demonstrated in the perioptic compartment between the optic canal and the anterior segment. However, regardless of the site of measurement along the optic nerve, there is a linear relationship between the perioptic CSF pressure and the ICP. A detailed discussion of the optic tracts, optic radiation and visual cortex is beyond the scope of this article.

\section{Ultrasonographic visualization of the optic nerve}

The introduction of high-frequency transducers and ultrasound units with a spatial resolution of 0.4 $\mathrm{mm}$ allowed the measurement of retrobulbar ONS. The patients are placed in a supine position and a thick layer of gel is applied on the upper closed eyelid and neutral patients' gaze. Two measurements are taken for each optic nerve: one in the transverse plane, with the probe being horizontal, and one in the sagittal plane, with the probe being vertical. The final ONSD is the mean of these measurements. The ONSD is measured $3 \mathrm{~mm}$ behind the optic disc. ${ }^{12,13}$

The globe is the dominant structure, with two compartments (anterior and posterior chamber). The anterior chamber is echofree and is delineated posteriorly by the strong reflecting line of the iris. The pupil appears as a translucent disruption of iris continuity. Posterior to it lays the anechoic lens. The anterior margin of the lens is not apparent, and neither is the posterior chamber, which is too thin to be visible. The lens diameter is $10 \mathrm{~mm}$ with a maximal thickness of $3-4 \mathrm{~mm} \cdot{ }^{12,13}$ The posterior margin of the lens is convex towards the vitreous cavity and, due to variable spatial relationship with the ultrasonic beam, is only partly apparent. The ciliary body produces a focal thickening of the eye wall, next to the margins of the lens. The vitreous humor is echofree, homogeneous and occupies more than two thirds of the eye ball volume. Since it only adheres to the posterior wall in a few points, movement of the vitreous humor relative to the wall can be observed during real time-scanning. ${ }^{12,13}$

The posterior wall of the eyeball is echogenic, often with no inner layers. With high frequency transducers and lowering of distal gain compensation, the choroid 
appears less echogenic than neighboring retina or sclera. Behind the eyeball, the intraconal fat pad is hyperechoic, mainly due to acoustic enhancement in the vitreous humor. The optic nerve appears as a sagittal hypoechoic structure, 4,5-5 mm thick, than runs from the outer part of the eyeball to the tip of the orbit. The length of the optic nerve is approximately $2,5 \mathrm{~cm}$. The optic disc is seen as a hyperechoic line in the posterior pole of the globe. It has a good interobserver variability, with a median difference of $0.2-0.3 \mathrm{~mm} .{ }^{12}$

An interesting additional finding is that the optic nerve sheath diameter does not change with patient positioning. The Trendelenburg position is often used in hypotensive patients, and the reverse Trendelenburg position $\left(30^{\circ}\right.$ head up) is often used in patients with head injury to help decrease intracranial pressure. According to Foster et al. ${ }^{13}$ the optic nerve sheath diameter measured by sonography in healthy individuals does not change appreciably with the Trendelenburg or reverse Trendelenburg position in comparison with the supine baseline.

Ultrasonography of the eye and orbit is not difficult to perform. However, thorough knowledge of ultrasound anatomy and scanning technique is mandatory for proper usage of the technique in the appropriate clinical setting. ${ }^{12,13}$

\section{Raised ICP and tomographic characteristics}

The ability of CT scanning to predict the presence or absence of intracranial hypertension has been debated in the literature. Attempts at predicting ICP in patients who present with abnormal head CT scans have not been as successful. ${ }^{3}$

Sadhu et al. ${ }^{14}$ demonstrated no increase in ICP associated with a normal head CT scan, poor correlation with slit ventricles and increased ICP, and high correlation with dilation of contralateral temporal horn and increased ICP. In 1981, Kishore et al. ${ }^{9}$ focused on hemorrhagic lesions and found that 55\% developed intracranial hypertension. Tabaddor et al. ${ }^{15}$ demonstrated a year later that ventricular compression correlated well with the level of ICP and that midline shift correlated poorly.

Lobato et al. ${ }^{16}$ demonstrated that patients with normal CT scans in the week posttrauma were unlikely to develop intracranial hypertension. However Eisenberg et al. ${ }^{17}$ demonstrated that $10 \%$ to $15 \%$ of patients with a normal initial head CT scan developed intracranial hypertension.

In 1992, Marshall et al..$^{18}$ introduced a classification system based on computed tomography findings. This system grouped patients with TBI into six groups based upon multiple CT characteristics. The patients were differentiated based on the presence or absence of a focal mass lesion, and diffuse injuries were subdivided into four groups based on CT signs suggestive of increased intracranial pressure (compression of basal cisterns and midline shift).

In an effort to estimate initial ICP in severe head injury patients many authors incorporated multiple findings from initial $\mathrm{CT}$, including the appearance of the cisterns, subdural hematoma size, intracerebral hematoma size, ventricular size, degree of subarachnoid hemorrhage, degree of contusion, the magnitude of midline shift, and the ventricular index. Approximately $80 \%$ of the estimated ICP were within $10 \mathrm{mmHg}$ of the measured ICP, but there was a significant discrepancy when the measured ICP exceeded $40 \mathrm{mmHg}$. Attempting to create a simpler prediction model incorporating ventricle size, sulci size, degree of transfalcine herniation, and gray/white matter differentiation, Miller et al. ${ }^{19}$ were unable to establish a significant correlation with ICP in patients with traumatic brain injury (TBI).

A general limitation of radiological interpretation is that TBI is a dynamic process and CT scan gives us a momentary picture. So serial CT scanning is indicated in different clinical settings. Patients with severe head injury should be reexamined frequently, as $25 \%$ of those patients with a normal head CT initially will demonstrate abnormalities on the follow-up scan. ${ }^{20}$ Two-thirds of patients deteriorating after 48 hours will have new hemorrhagic lesions, either intra- or extraaxial. These delayed lesions are associates with a poorer outcome; however prognosis is improved by earlier recognition and treatment. ${ }^{20}$

Despite earlier studies used CT scanning as an indicator of raised ICP, actually to provide more accurate results the invasive ICP should be used.

\section{Raised ICP and optic nerve sheat enlargement}

When ICP is normal the optic nerve sheat diameter remains the same as baseline. When it rises the CSF flows towards into perineural subarachnoid space and increases the pressure around the optic nerve. ${ }^{12}$ This results in expansion of the dural sheath and an increase in the diameter of the ONS. ${ }^{12,13}$ The assymetrical distribution of trabecular fibers along the subarachnoid space and the fact that the retrobulbar segment of the sheat is the thinnest along the nerve are the anatomical explanations why the expansion mainly affects the anterior segment of the dural sheath $3 \mathrm{~mm}$ behind the globe. In 
contrast, the posterior regions exhibit markedly less or no obvious dilation. ${ }^{12}$ Hansen and Helmke ${ }^{21}$ showed that a segment of the optic nerve sheath diameter approximately $3 \mathrm{~mm}$ behind the papilla showed maximal diameter fluctuations induced by gelatinous injections in postmortem preparations. This diameter landmark has been used in several clinical trials correlating elevated CSF pressure-derived intracranial pressure with optic nerve sheath diameter-derived intracranial pressure.

Most authors have suggested that the upper normal value of ONSD is $5 \mathrm{~mm}$, however latter studies suggest that the cut-off value of ONSD that provides the best accuracy for the prediction of intracranial hypertension ( ICP $=20 \mathrm{mmHg}$ ) is $5.7-6.0 \mathrm{~mm}$, and that ONSD values above this threshold should alert the clinician to the presence of raised ICP ${ }^{11,13,21}$ (Figure 1).

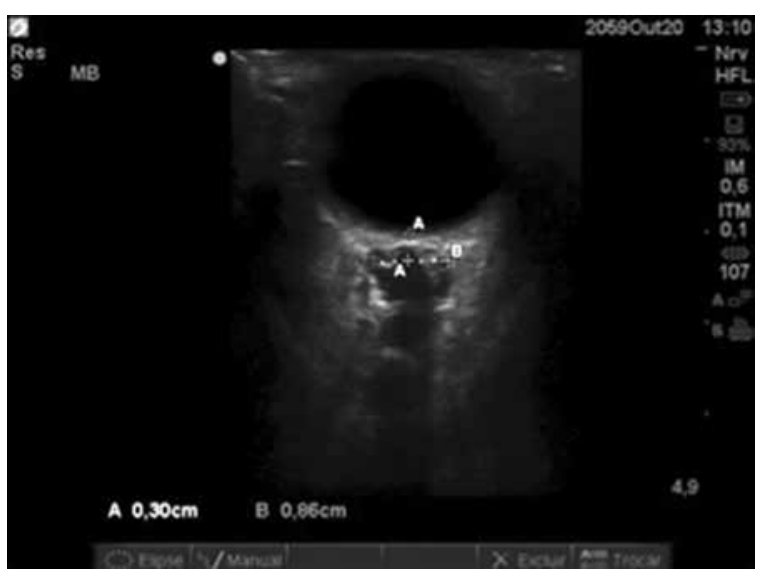

Figure 1 - Optic nerve sheat diameter (raised ICP).

According to Geeraerts et al., ${ }^{22}$ a strong relationship was found between the mean ONSD and ICP. The negative likelihood ratio of the $5.8 \mathrm{~mm}$ cut-off value shows a very low probability of having high ICP when ONSD was less than this. A looser relationship was found between OND and ICP. Others authors have found the same relations. The variation of ONSD during raised ICP cannot therefore be related to optic nerve dilatation (as during optic nerve edema), but to its sheath distension, due to the increase in CSF pressure surrounding the optic nerve. There are instances of stably high ICP and rapid raises of ICP are accompanied by increases in the optical nerve sheath diameter, assessed by ultrasound, from $5.5 \mathrm{~mm}$ to $7 \mathrm{~mm}$. Considering these measurements the sensitivity lie in the range of $87 \%-95 \%$ and specificity in the range of $79 \%-100 \% .{ }^{22}$

Cases of unilateral papilloedema and asymmetrical dilations of perineural sheat have been reported. Although, for confirmation purposes the sonographic measurement of the ONSD should be performed bilaterally. ${ }^{22}$
There is also evidence the enlargement of ONDS is a dynamic process which varies according to changes in the ICP. It should be observed with caution, since the correction of increased ICP does not follow the same kynematic in reduction of optic nerve sheat diameter. ${ }^{13}$

Despite of the advantages, the optic nerve sheat ultrasonography has some limitations. In patients with ocular trauma and other diseases of optic nerve complex the assessment of ONSD can be very difficult. Traumatic optic neuropathy is seen in a significant number of patients with severe head injury and the effects of eye trauma on ONSD are unclear. ${ }^{13}$ Additionally, optic nerve enlargement can occur due to secondary involvement from a variety of orbital and systematic abnormalities such as tumour, inflammation, Grave's disease, sarcoidosis, pseudotumour, metastasis and haemorrhage in and around the optic nerve complex as well as hydrops from extrinsic tumour. As with most ultrasound examinations, the quality of optic nerve sonography relies heavily on the skill and diligence of the observer. ${ }^{12}$

Standardisation of the examination is of great importance since measurements of the ONSD are themselves very small and any alterations approach the intrinsic accuracy limits of the ultrasound equipment. Inappropriate technique may lead to significant errors and reduce the benefit of the method. Common pitfalls include inadequate depiction of the optic nerve in the axial plane, inaccurate designation of the sheath contours and erroneous placement of the cursors. It is therefore mandatory that trained personnel perform all examinations only. ${ }^{12,13}$

\section{Raised ICP and transcranial Doppler sonography}

The possibility of studying cerebral hemodynamics with Doppler ultrasound, reported for the first time in 1982 by Aaslid et al. ${ }^{23}$ marked the beginning of a new era for clinical evaluation of cerebral blood flow (CBF) autoregulation. The use of TCD as a marker of ICP was initially described by Klingelhöfer $e t$ al. ${ }^{24}$ in a pilot study changes in ICP were compared with the TCD findings of the MCA. Data from five patients with brain death showed that changes in the ICP influenced the flow patterns considerably. These changes could be recorded quantitatively by means of the PI and the mean FV. Increases in ICP were accompanied by an increase in PI (owing to a decrease in diastolic and mean velocities). With increasing ICP, there was a progressive reduction in the MFV and EDV with an increase in RI. The authors subsequently demonstrated an association between ICP and flow patterns in a subgroup of neurosurgical patients with cerebral hypertension. ${ }^{24}$ 
According to Behrens et al. ${ }^{25}$ the assessment of the pulsatility index $(\mathrm{PI})$ correlated well with ICP $(\mathrm{r}=0.94)$, indicating its usability as a method to noninvasively assess ICP. However others do not support measurement of intracranial blood flow velocity to determine ICP. Computer simulations demonstrate that this shortcoming may be attributable to individual variation in physiological parameters that may be associated with disease, but could be related to normal variation as well. The variability of the in vivo ICP-PI relationship in previous studies, does not support TCD as a tool to provide reliable information on ICP. Despite this data TCD may have a role in situations where the indication of invasive monitoring is not clearly established: stroke, paediatric cases, liver failure, minor head injury assessed on accident and emergency wards and preeclampsia. ${ }^{25}$

\section{Magnetic resonance image and intraranial hypertension}

According to MRI using ONSD measurement is potentially useful in detecting raised ICP. ONSD (but not $\mathrm{OND}$ ) is strongly related to ICP, a finding that reflects distension of the nerve sheath during increases in CSF pressure. On T2-weighted sequences, water (and CSF) exhibits a high signal (white). Fat and grey matter appear as light grey, and white matter as dark grey. The perioptic CSF is surrounded by orbital fat. Contrast between CSF and orbital fat can be improved with fat suppression, increasing the image resolution for the ONSD measurement.

The most useful clinical message derived from current data may be the following thresholds; an ONSD less than $5.30 \mathrm{~mm}$ is unlikely to be associated with raised ICP, and an ONSD above $5.82 \mathrm{~mm}$ indicates that the probability of raised ICP is $90 \%$.

A major limitation of the method is probably related to MRI by itself, with limited access, necessity of patient transfer in a magnetic area and specific contraindications, especially in the acute phase after trauma. ${ }^{26}$

\section{Fundoscopic findings}

Fundoscopic evidence of papilloedema can provide useful evidence of intracranial hypertension in cases of chronic raised ICP.

When ICP increases, the pressure in the ONS increases linearly, which distends the nerve sheat. The increased pressure results in stasis of axoplasmic transport, which is thought to cause papilledema. Because of the close relationship between ICP and papilledema, examination of the optic nerve with an ophthalmoscope can estimate ICP. The Frisen scale grades the severity of papilledema on examination, with grade 0 being normal and grade 5 being the most severe. According to Frisén, ${ }^{27}$ who have published the initial study shows a sensitivity of $93 \%-100 \%$ and a specificity of $88 \%-96 \%$ for the detection of papilledema from optic nerve photograph.

In contrast, experimental studies clearly show that edema of the optic disk requires a few days to develop and resolve. Another limitation is that medical practitioners lack confidence in their ability to do a fundoscopic examination. According to Steffen et al. ${ }^{28}$ even when the nerves can be examined reliably, the absence of papilledema does not preclude the presence of ICP elevation.

Another aspect of the optic nerve examination used to approximate ICP is spontaneous venous pulsations. Levin ${ }^{29}$ has shown that when the pulsations are present, the ICP must be less than $190 \mathrm{mmH}_{2} \mathrm{O}$. However, SVPs are absent in $10 \%$ of normal people, so a lack of pulsations does not always indicate increased ICP.

This data make the ophtalmoscopy a less useful clinical sign in settings where there may be acute increases, dynamic changes in ICP, lack of spontaneous venous pulsations or incongruence in the fundoscopic examination.

\section{Discussion}

Intracranial pressure (ICP) monitoring is essential in severe traumatic brain injury (TBI) but requires a cranial trephination and, when intraparenchimal probes are used, brain tissue penetration. It is therefore an invasive, potentially dangerous, and obviously cumbersome method, which must be reserved for patients at risk of raised ICP.

In recent years, emergency medicine has seen the growth and expansion of emergency ultrasound (US). This expansion has been in the form of not only the proliferation of ultrasound technology to more emergency departments, but also the expansion of different emergency ultrasound applications. The ability to predict whether or not ICP is elevated on the basis of CT scan findings would aid in defining which patients would benefit from more invasive monitoring, allow for more rapid treatment, and conserve precious neurosurgical resources..$^{30}$ It is well known that patients with severe TBI are at risk for intracranial hypertension. When associated with reduced cerebral perfusion pressure (CPP) there is a correlation with worse outcomes. ${ }^{30}$ The current Brain Trauma Foundation recommendations for ICP monitoring (level II) are to place probes in all salvageable patients with GCS between 3-8 (after resuscitation) and abnormal CT scan. Additionally ICP monitoring 
(level III) may be indicated in patients with normal CT scan if two or more of the following are noted on admission: age over 40 years, motor posturing (unilateral or bilateral), or systolic blood pressure $<90 \mathrm{mmHg}{ }^{3}$

The ocular nerve is part of the central nervous system and in case of raised pressure in the cerebrospinal fluid its sheath inflates. Association between increases in optical nerve sheath diameter and intracranial hypertension has been previously confirmed. When needed, ocular ultrasound therefore can be rapidly performed at the patient's bedside and help to identify conditions of normal and high ICP or rapid changes in ICP. There are some discrepancies between the authors and which is the cutoff value of sheath diameter remains to be identified to allow for a more accurate diagnosis. ${ }^{11-13}$

It should also be noted that, with raised ICP, the optic nerve diameter increases initially and then leads to papilloedema, so transorbital sonography is able to show increased ICP earlier than ophthalmoscopy. ${ }^{13,22}$

Ocular sonography is believed to be safe. However it has been suggested that exposure to ultrasound might cause tissue damage by heating and cavitation. In the United States the FDA implemented an output intensity limit for all eye exposures $\left(50 \mathrm{~mW} / \mathrm{cm}^{2}\right)$, which is 12 times lower than that allowed for general soft tissue exposure. In the Europe sonographers are advised to keep ultrasound output power as low as reasonably achievable without compromising the diagnostic value of the examination. Where low values cannot be achieved, examination times should be kept as short as possible. ${ }^{12,13}$

Whilst it is unlikely that TCD will replace invasive ICP monitoring in the near future, it may have a role in situations where the role of invasive monitoring is not clearly established: stroke, paediatric cases, liver failure, minor head injury assessed on accident and emergency wards and preeclampsia. ${ }^{25}$

MRI can be used to determine the amount of subarachnoid fluid around the optic nerve and it reliably detects the optic nerve and sheath size. In patients with papilledema, the subarachnoid space dilates, the optic nerve is compressed, and the nerve sheath is widened. The precision of MRI seems to be higher than that of ultrasound. ${ }^{26}$

\section{Conclusion}

The authors of the present review strongly support the need of continuous invasive ICP measurement in neuro-ICU patients with suspected raised ICP. Cranial CT scan followed by insertion of an intraparenchymal probes remains the gold standard for ICP monitoring in head injuried patients. Ocular sonography could serve as a bedside and easy-to-use detection tool for raised ICP. Our objective is to expose some non-invasive methods to predict the risk of intracranial hypertension and select patients to ICP monitoring, especially in those with normal CT scans.

\section{References}

1. Sosin DM, Sniezek JE, Waxweiler RJ. Trends in death associated with traumatic brain injury, 1979 through 1992. Success and failure. JAMA. 1995;273(22):1778-80.

2. Barr R, Gean A. Craniofacial trauma. In: Brant W, Helms $\mathrm{C}$, editors. Fundamentals of diagnostic radiology. 2nd ed. Philadelphia: Lippincott Williams \& Wilkins; 1999. p. 49.

3. The Brain Trauma Foundation. The American Association of Neurological Surgeons. The Joint Section on Neurotrauma and Critical Care. Guidelines for cerebral perfusion pressure. J Neurotrauma. 2000;17(6-7):507-11.

4. Palmer S, Bader MK, Qureshi A, Palmer J, Shaver T, Borzatta $M$, et al. The impact on outcomes in a community hospital setting of using the AANS traumatic brain injury guidelines. Americans Associations for Neurologic Surgeons. J Trauma. 2001;50(4):657-64.

5. Arabi YM, Haddad S, Tamim HM, Al-Dawood A, Al-Qahtani $\mathrm{S}$, Ferayan A, et al. Mortality reduction after implementing a clinical practice guidelines-based management protocol for severe traumatic brain injury. J Crit Care. 2010;25(2):190-5.

6. Cremer OL, Van Dijk GW, Van Wensen E, Brekelmans GJ, Moons KG, Leenen LP, et al. Effect of intracranial pressure monitoring and targeted intensive care on functional outcome after severe head injury. Crit Care Med. 2005;33(10):2207-13.

7. Shafi S, Diaz-Arrastia R, Madden C, Gentilello L. Intracranial pressure monitoring in brain-injured patients is associated with worsening of survival. J Trauma. 2008;64(2):335-40.

8. Chesnut RM. Intracranial pressure monitoring in braininjured patients is associated with worsening of survival. J Trauma. 2008;65(2):500-1.

9. Kishore PR, Lipper $\mathrm{MH}$, Becker DP, Domingues da Silva AA, Narayan RK. Significance of CT in head injury: correlation with intracranial pressure. AJR Am J Roentgenol. 1981;137(4):829-33.

10. Gentry LR. Anatomy of the orbit. Neuroimaging Clin N Am. 1998;8(1):171-94.

11. Liu D, Kahn M. Measurement and relationship of subarachnoid pressure of the optic nerve to intracranial pressures in fresh cadavers. Am J Ophthalmol. 1993;116(5):548-56.

12. Dudea SM. Ultrasonography of the eye and orbit. Med Ultrason. 2011;13(2):171-4.

13. Foster T, Tayal VC, Saunders T, Norton J. Emergency ultrasound optic nerve sheat measurement to detect increased intracranial pressure in head injury patients: preliminary study of interobserver variability in normal human subjects. Acad Emerg Med. 2003;10:487-8.

14. Sadhu VK, Sampson J, Haar FL, Pinto RS, Handel SF. Correlation between computed tomography and intracranial pressure monitoring in acute head trauma patients. Radiology. 1979;133(2):507-9. 
15. Tabaddor K, Danziger A, Wisoff HS. Estimation of intracranial pressure by CT scan in closed head trauma. Surg Neurol. 1982;18(3):212-5.

16. Lobato RD, Sarabia R, Rivas JJ, Cordobes F, Castro S, Muñoz MJ, et al. Normal computerized tomography scans in severe head injury. Prognostic and clinical management implications. J Neurosurg. 1986;65(6):784-9.

17. Eisenberg HM, Gary HE Jr, Aldrich EF, Saydjari C, Turner $B$, Foulkes MA, et al. Initial CT findings in 753 patients with severe head injury. A report from the NIH Traumatic Coma Data Bank. J Neurosurg. 1990;73(5):688-98.

18. Marshall LF, Marshall SB, Klauber MR, Van Berkum Clark $M$, Eisenberg $\mathrm{H}$, et al. The diagnosis of head injury requires a classification based on computed axial tomography. $\mathrm{J}$ Neurotrauma. 1992;9(Suppl 1):S287-92.

19. Miller MT, Pasquale M, Kurek S, White J, Martin P, Bannon K, et al. Initial head computed tomographic scan characteristics have a linear relationship with initial intracranial pressure after trauma. J Trauma. 2004;56(5):967-72.

20. Howells TP, Piper IR, Jones PA, Souter M, Miller JD. Design of a research database for the study of secondary insults following head injury. J Neurotrauma. 1995;12:471.

21. Hansen HC, Helmke K. Validation of the optic nerve sheath response to changing cerebrospinal fluid pressure: ultrasound findings during intrathecal infusion tests. J Neurosurg. 1997;87(1):34-40.

22. Geeraerts T, Launey Y, Martin L, Pottecher J, Vigué B, Duranteau $\mathrm{J}$, et al. Ultrasonography of the optic nerve sheath may be useful for detecting raised intracranial pressure after severe brain injury. Intensive Care Med. 2007;33(10):1704-11.

23. Aaslid R, Markwalder TM, Nornes H. Noninvasive transcranial Doppler ultrasound recording of flow velocity in basal cerebral arteries. J Neurosurg. 1982;57(6):769-74.
24. Klingelhöfer J, Conrad B, Benecke R, Sander D, Markakis E. Evaluation of intracranial pressure from transcranial Doppler studies in cerebral disease. J Neurol. 1988;235(3):159-62.

25. Behrens A, Lenfeldt N, Ambarki K, Malm J, Eklund A, Koskinen LO. Transcranial Doppler pulsatility index: not an accurate method to assess intracranial pressure. Neurosurgery. 2010;66(6):1050-7.

26. Geeraerts T, Newcombe VF, Coles JP, Abate MG, Perkes IE, Hutchinson PJ, et al. Use of T2-weighted magnetic resonance imaging of the optic nerve sheath to detect raised intracranial pressure. Crit Care. 2008;12(5):R114.

27. Frisén L. Swelling of the optic nerve head: a staging scheme. J Neurol Neurosurg Psychiatry. 1982;45(1):13-8.

28. Steffen H, Eifert B, Aschoff A, Kolling GH, Völcker HE. The diagnostic value of optic disc evaluation in acute elevated intracranial pressure. Ophthalmology. 1996;103(8):1229-32.

29. Levin BE. The clinical significance of spontaneous pulsations of the retinal vein. Arch Neurol. 1978;35(1):37-40.

30. Tayal VS, Neulander M, Norton HJ, Foster T, Saunders $\mathrm{T}$, Blaivas M. Emergency department sonographic measurement of optic nerve sheath diameter to detect findings of increased intracranial pressure in adult head injury patients. Ann Emerg Med. 2007;49(4):508-14.

Correspondence address

Leonardo Christian Welling

Division of Neurological Surgery, Hospital das Clínicas,

University of Sao Paulo

Rua Dr. Enéas de Carvalho Aguiar, 255, Jardim Paulista

05403-000 - São Paulo, SP, Brazil

Telephone: (42) 9121-4600

E-mail: leonardowelling@yahoo.com.br 